\title{
Hollow Cathode and Low-Thrust Extraction Grid Analysis for a Miniature Ion Thruster
}

\author{
Richard Wirz, ${ }^{1}$ Regina Sullivan, ${ }^{2}$ JoHanna Przybylowski, ${ }^{2}$ and Mike Silva ${ }^{2}$ \\ ${ }^{1}$ Propulsion and Materials Section, Jet Propulsion Laboratory, California Institute of Technology, Pasadena, CA 91109, USA \\ ${ }^{2}$ Aeronautics, California Institute of Technology, Pasadena, CA, USA
}

Correspondence should be addressed to Richard Wirz, richard.e.wirz@jpl.nasa.gov

Received 3 January 2008; Revised 20 April 2008; Accepted 23 June 2008

Recommended by Paul K. Chu

\begin{abstract}
Miniature ion thrusters are well suited for future space missions that require high efficiency, precision thrust, and low contamination in the $\mathrm{mN}$ to sub- $\mathrm{mN}$ range. JPL's miniature xenon Ion (MiXI) thruster has demonstrated an efficient discharge and ion extraction grid assembly using filament cathodes and the internal conduction (IC) cathode. JPL is currently preparing to incorporate a miniature hollow cathode for the MiXI discharge. Computational analyses anticipate that an axially upstream hollow cathode location provides the most favorable performance and beam profile; however, the hot surfaces of the hollow cathode must be sufficiently downstream to avoid demagnetization of the cathode magnet at the back of the chamber, which can significantly reduce discharge performance. MiXI's ion extraction grids are designed to provide $>3 \mathrm{mN}$ of thrust; however, previous to this effort, the low-thrust characteristics had not been investigated. Experimental results obtained with the MiXI-II thruster (a near replica or the original MiXI thruster) show that sparse average discharge plasma densities of $\sim 5 \times 10^{15}-5 \times 10^{16} \mathrm{~m}^{-3}$ allow the use of very low beamlet focusing extraction voltages of only $\sim 250-500 \mathrm{~V}$, thus providing thrust levels as low as $0.03 \mathrm{mN}$ for focused beamlet conditions. Consequently, the thrust range thus far demonstrated by MiXI in this and other tests is $0.03-1.54 \mathrm{mN}$.
\end{abstract}

Copyright (C) 2008 Richard Wirz et al. This is an open access article distributed under the Creative Commons Attribution License, which permits unrestricted use, distribution, and reproduction in any medium, provided the original work is properly cited.

\section{INTRODUCTION}

\subsection{Background and motivation}

Ion thrusters are well known for their ability to deliver continuous thrust at high efficiency $\left(\eta_{\text {tot }} \sim 60-70 \%, \eta_{\mathrm{ud}} \sim 80-\right.$ $90 \%)$ and high specific impulse ( $I_{\text {sp }} \sim 2000-5000$ seconds) with the use of benign propellants (e.g., xenon and argon). A miniature ion thruster that can provide these performance advantages at a maximum thrust near $3 \mathrm{mN}$ and minimum thrust $<0.1 \mathrm{mN}$ is attractive for a variety of future space missions [1]. Candidate mission include many of NASA's and ESA's Exoplanet finding missions which require precision control of large spacecraft and formation flying as well as Earth-orbiting missions that require precision orbit control.

Historically, miniaturization of ion thruster technologies has presented many design and performance challenges that have put the concept out of reach for mission designers. However, recent advancements have shown that reasonable performance is possible for small ion thrusters if the development and validation of key technologies are continued.
The miniature xenon ion (MiXI) thruster, shown in Figure 1, is a significantly scaled-down version of conventional ion thrusters that has demonstrated an efficient discharge and a high-performance ion extraction grid assembly [2]. Similarly to conventional ion thrusters, the MiXI thruster uses electrons released from the cathode to create a low-density $\left(\sim 10^{17} \mathrm{~m}^{-3}\right)$ xenon plasma. The xenon propellant can be introduced to the chamber through the back flange or through the cathode if a hollow cathode is used. A 3ring cusp magnetic field is used to confine the plasma and improve discharge efficiency. Plasma ions are accelerated out of the thruster at high velocities $(\sim 40,000 \mathrm{~m} / \mathrm{s})$ through the ion extraction grid assembly by an applied thruster voltage of up to $1 \mathrm{kV}$, thus providing high specific impulse of over 3000 seconds. The physical transparency of the screen grid is 0.67 and during ion extraction the transparency is approximately $0.72-0.75$ for the conditions discussed herein. Additional details on the thruster configuration and operation are given in Sections 2 and 3. Previous studies and performance tests with the MiXI have used filament cathodes and the internal conduction (IC) cathode [2-4]. The IC cathode 


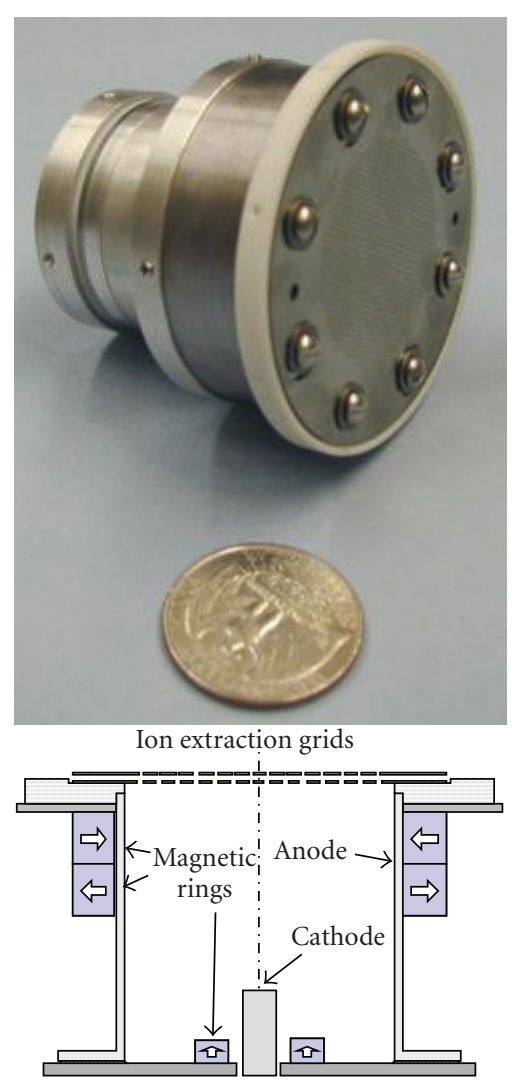

FIgUre 1: Miniature xenon ion (MiXI) thruster with basic schematic.

demonstrated high performance but it is a risky technology for ion thrusters due to its low maturity; therefore miniature hollow cathodes were designed and built specifically for the MiXI thruster. In future experimental investigations we will use one of these cathodes for the thruster discharge and, as evident from other ion thruster discharge studies, the axial location of the cathode has a strong effect on thruster performance. Therefore, in this study, we use an ion thruster discharge model, DC-ION, to help determine a favorable axial position of the hollow cathode within the discharge before we position the cathode inside the thruster for testing [5].

Another important aspect of the MiXI design is the miniature ion extraction grids. The MiXI grids are designed to achieve thrust up to $\sim 3.0 \mathrm{mN}$, and have thus far demonstrated high performance at nominal thrust levels [4]. Since MiXI is considered an attractive candidate for missions that require high precision and a relatively large thrust range, this study investigates the minimum continuous thrust that MiXI can achieve with the current grid set in an effort to find the minimum thrust available for future candidate missions.

\subsection{Objective}

In Section 2 computational analysis is used to determine the optimal location of the hollow cathode in MiXI's discharge chamber. In Section 3 the MiXI ion extraction grid assembly is experimentally and computationally characterized in the low-thrust regime to determine the minimum thrust capability of the grids.

\section{SENSITIVITY ANALYSIS FOR EMPLOYING A HOLLOW CATHODE IN MiXI'S DISCHARGE CHAMBER}

In this section JPL's DC-ION computational model is used to anticipate the performance of the MiXI thruster for a range of hollow cathode axial locations and cathode magnet strengths. These results will guide future experiments in which MiXI's miniature hollow cathodes will be used as the discharge and neutralizer electron sources.

\subsection{Miniature, low-flow hollow cathodes}

Previous investigations show that attractive performance is possible for the MiXI thruster with low-power electron sources such as the hollow cathodes used in conventional ion thrusters. Hollow cathodes are a mature technology and typically require less power per amp of emitted electrons than other discharge cathode options. Cathodeless discharge options, such as the microwave and rf discharges discussed in [3], will be investigated for the MiXI thruster if the high efficiency of cathode discharges is not achieved with cathode options. Conventional-sized hollow cathodes require a designated propellant feed $(>1 \mathrm{sccm})$ that can exceed the propellant needs for the entire MiXI discharge chamber (typically $0.05-0.5 \mathrm{sccm}$ ), which, may result in very low efficiencies and poor throttleability [3]. Previous investigations into small hollow cathodes with a $1.22 \mathrm{~mm}$ insert ID have shown that scaling down to the discharge currents and propellant flow rates of the MiXI thruster $\left(J_{D} \sim 50-500 \mathrm{~mA}\right.$ and $0.2-1.0 \mathrm{sccm}$, resp.) is difficult if the cathode is to run efficiently in self-heated mode [5-7]. To address this challenge, JPL designed and built two miniature hollow cathodes (Figure 2) specifically for MiXI's low-flow and current conditions. These hollow cathodes are a scaleddown version of conventional hollow cathodes. They are designed to have sufficiently high current capacity so that they may scale to larger ion thruster sizes $(>3 \mathrm{~cm})$ and small Hall thrusters, and may also provide a reliable and efficient option for neutralizing the plumes of other small electric propulsion thrusters such as electrospray (i.e., colloid and FEEP) thrusters.

\subsection{Hollow cathode axial location analysis}

In this study, JPL's ion thruster model (DC-ION) simulates MiXI thruster performance at a range of axial locations of a miniature hollow cathode inside the discharge. For ring cusp discharges of this orientation, the axial placement of the cathode (for a given magnetic field geometry) can drastically change the paths the electrons take to the anode and hence the quality of the electron confinement; which, in turn, can greatly affect ionization efficiency and performance. Details of the DC-ION model and hollow cathode simulations with DC-ION are given in $[4,8,9]$. For this analysis, the entire 


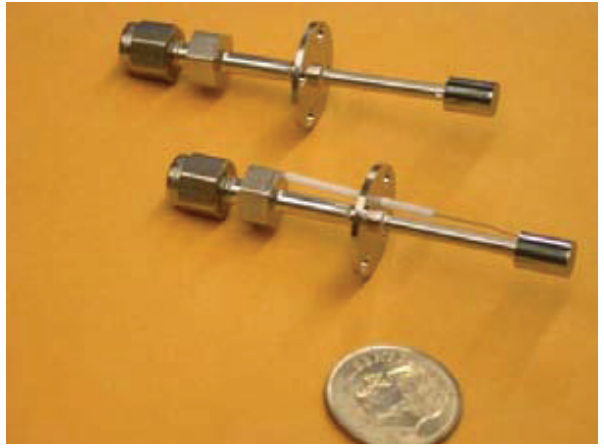

FIgURE 2: MiXI's miniature hollow cathodes.

discharge propellant flow is assumed to come from the hollow cathode. The primary electrons produced from the hollow cathode exit are assumed to exhibit an accelerated half-Maxwellian velocity distribution as used for the hollow cathode input for the NSTAR thruster in [5]. At this time, the hollow cathode is assumed to operate at similar voltages and electron tempe ratures as the NSTAR hollow cathode at TH12, resulting in the model inputs shown in Table 1 for nominal MiXI operation.

The DC-ION discharge model was used to compare the propellant efficiency and beam flatness for several hollow cathode orifice plate and grid separation distances; which are shown as " $x$ " in Figure 3 and are normalized by the discharge length "L." Results from the DC-ION analysis shown in Figure 4 suggest that an upstream cathode location ion thrusters yields the best propellant efficiency and beam profile for nominal MiXI operation. Upstream cathode placements are also used in larger ion thrusters to increase the field-free region available for ionization. One must be careful to place the cathode orifice beyond the magnetic maximum of the cathode magnet ring to avoid magnetic mirror effects; therefore, the maximum $x / L$ (i.e., 0.67 ) is forward of the local cathode ring maximum in the MiXI discharge. Moving the cathode further upstream (i.e., $x / L>$ 0.67 ) is also undesirable since the cathode heat can possibly demagnetize the cathode magnets, as discussed later. Figure 5 gives the beam profiles for all the configurations investigated herein and shows that the beam profile progressively peaks on-axis as the hollow cathode is located closer to the extraction grids. This behavior for closer spacing is due to the fact that the electrons emitted by the cathode have a smaller distance to undergo a collision that will cause them to more readily diffuse away the thruster axis where they will create more ions off-axis, resulting in a flatter profile.

\subsection{Magnetic field analysis}

Currently, MiXI employs a relatively weak cathode magnet since favorable performance was found with filament discharges in previous efforts [2]; however, conventional ion thrusters (such as NASA's NSTAR thruster), which use hollow cathodes, typically use high cathode magnet strengths. Therefore, when MiXI uses a hollow cathode in the discharge, it may prove beneficial to strengthen the
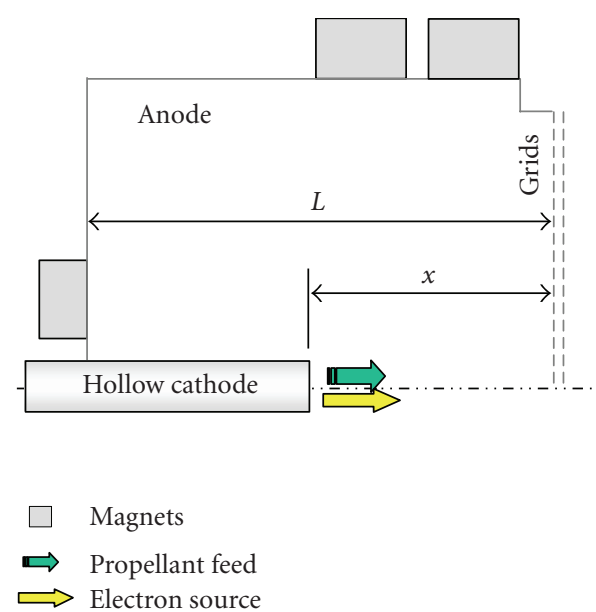

FIGURE 3: Basic MiXI configuration showing axial distance between cathode face and screen grid, $x$, and discharge chamber length, $L$.

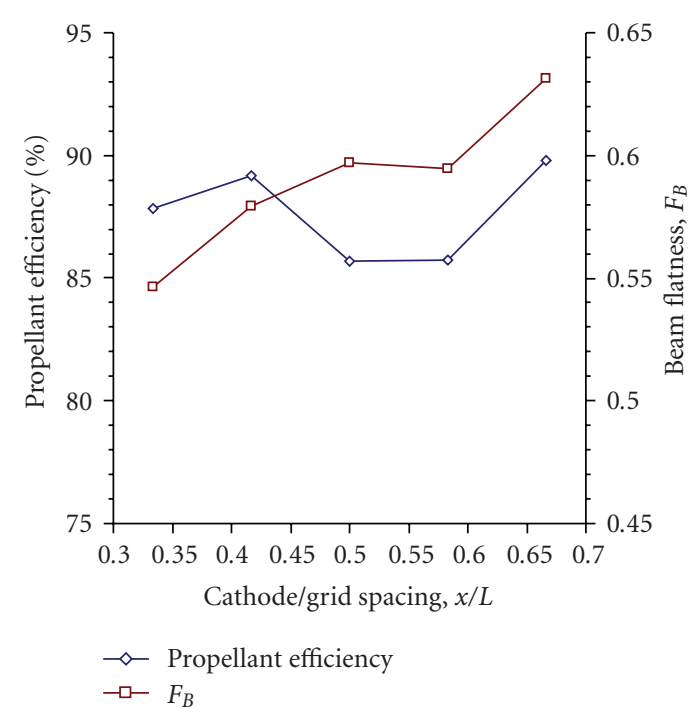

Figure 4: Anticipated MiXI performance versus Cathode/Grid spacing $(x / L)$ using baseline MiXI configuration. Results generated by DC-ION model.

cathode magnet as done in conventional ion thrusters. At the other extreme, due to the small size of the MiXI discharge, there is some concern that the heat from the hollow cathode may demagnetize the cathode magnet, especially if the cathode is positioned far upstream. To address these potential scenarios, DC-ION runs were used to simulate the impact of changing MiXI's cathode magnet strength. In the following analysis, "High Field" is the condition where cathode magnet strength is increased by a factor of two by doubling the dimension along the magnetization direction, while "Low Field" assumes the cathode magnet has been entirely demagnetized by cathode heat (in the computations the magnet strength was set to zero). Previous experimental results show that loss of the cathode magnet can reduce the discharge performance by over $10 \%$ [4]. Figure 7 shows the anticipated performance for the Baseline, High Field, and 
Table 1: MiXI Throttle Point THM1.

\begin{tabular}{cccccccc}
\hline Total flow $[\mathrm{sccm}]$ & $J_{D}[\mathrm{~mA}]$ & $V_{D}[\mathrm{~V}]$ & $J_{B}[\mathrm{~mA}]$ & $V_{B}[\mathrm{~V}]$ & $V_{A}[\mathrm{~V}]$ & \multicolumn{2}{c}{ Model specific inputs } \\
& & & & & $V_{p}[\mathrm{~V}]$ & $T_{p}[\mathrm{eV}]$ \\
\hline 0.29 & 280 & 25.0 & $\sim 20$ & 1000 & -200 & 20.0 \\
\hline
\end{tabular}

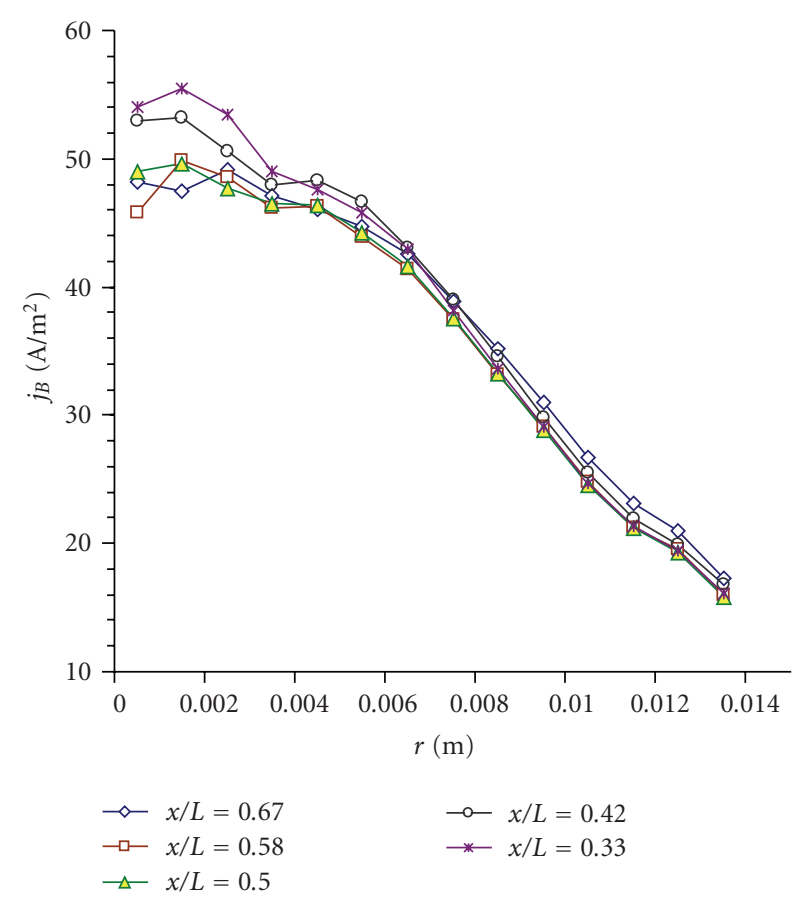

FIgure 5: Anticipated MiXI beam profiles versus Cathode/Grid spacing $(x / L)$ using baseline MiXI configuration. Results generated by DC-ION model.

Low Field configurations at cathode locations $x / L=0.42$ and 0.67 . These $x / L$ conditions were chosen since they give the two highest propellant efficiencies and represent downstream and upstream cathode positions, respectively. Figure 6 shows the beam profiles for these scenarios. As expected, the performance effects are most pronounced when the cathode is in the most upstream position (i.e., $x / L=0.67$ ), closest to the cathode magnet.

\section{LOW THRUST ANALYSIS FOR EXTRACTION GRIDS}

In this section a combination of experimental and computational investigations are used to assess the low thrust characteristics of the MiXI ion extraction grids.

\subsection{Ion extraction at low thrust levels}

The plasma sheath immediately upstream of the ion extraction grids will change shape based on the extractor geometry, voltages, and plasma properties. In general, for given upstream plasma properties and extractor geometry, the sheath will increase in size with increasing total voltage, $V_{T}$, to provide the space-charge current determined by the
Child-Langmuir law [10]. Figure 8 shows how the sheath adjusts at different total voltage values (assuming all other properties, such as upstream plasma density, are constant). In this figure $V_{\text {foc }}$ is the limit below which we observe direct ion impingement on the accel grid, and $V_{\text {xo }}$ is the voltage beyond which we observe cross-over impingement on the accel grid. The process of changing $V_{T}$ to achieve the nominal operating condition in Figure 8(a) is called "focusing." From this behavior one can also see that increasing the discharge chamber plasma density increases the current density at the screen hole plasma sheath, thus increasing $V_{\text {foc }}$ for a given grid geometry.

\subsection{Experimental testing apparatus}

A second lab model of the $3 \mathrm{~cm}$ diameter MiXI thruster "MiXI-II" for characterization of MiXI components, performance, and beam. MiXI-II is identical to the original 3-ring cusp MiXI thruster [1] except that the thruster mount (at the aft end of the thruster) is simplified and the grid mounts were modified to provide improved contact between the grids and the electrical leads. Figure 9 shows MiXI-II mounted in the vacuum chamber. As shown schematically in Figure 9, MiXI uses a 3-ring cusp discharge that is $3 \mathrm{~cm}$ in diameter with two ion extraction grids at the downstream end of the discharge. In this study, MiXI-II is used to analyze the grid performance at low thrust levels.

\subsubsection{MiXI's high-performance extraction grids}

A combination of experimental and computational analyses was used to design the MiXI grids [2]. These grids provide high performance since they employ a small hole accelerator grid (SHAG) design that exhibits low neutral transparency while still providing sufficient ion transparency to allow over $3 \mathrm{mN}$ of thrust. As described in [1], the current MiXI grid set was designed with relatively high grid thickness to provide longer grid life and lower susceptibility to thermal deflections relative to earlier designs. The maximum thrust capacity with the current MiXI grid set is $\sim 3 \mathrm{mN}$ (assuming a conservative beam flatness of 0.52 , typical MiXI beam flatness is over 0.6). In this analysis we investigate the lower thrust limit of the MiXI grid set by examining the direct impingement limits with computational and experimental analyses. For this investigation, MiXI-II uses filament cathodes for the neutralizer (shown in Figure 9) and discharge electron sources to provide a simple robust system for grid performance analysis. The use of a discharge filament cathode, as opposed to a hollow cathode, will have some effect on overall performance (increased cathode heater power and likely reduced propellant efficiency) but 


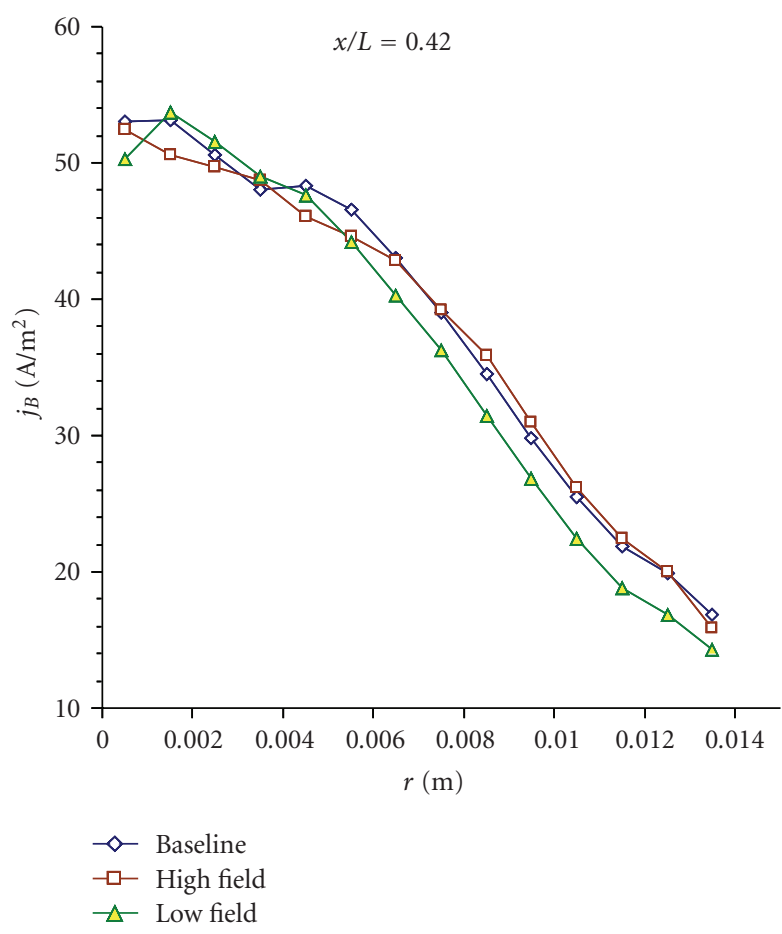

(a)

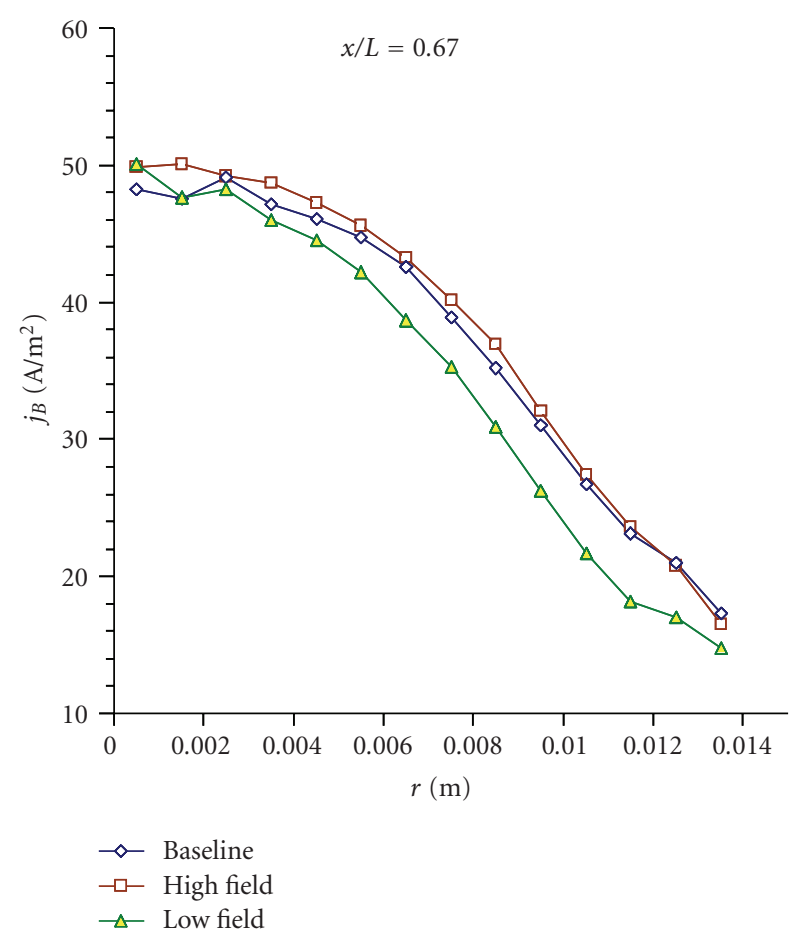

(b)

FIGURE 6: Anticipated MiXI thruster performance versus Cathode/Grid spacing $(x / L)$ at different cathode magnet strengths. Results generated by DC-ION model.

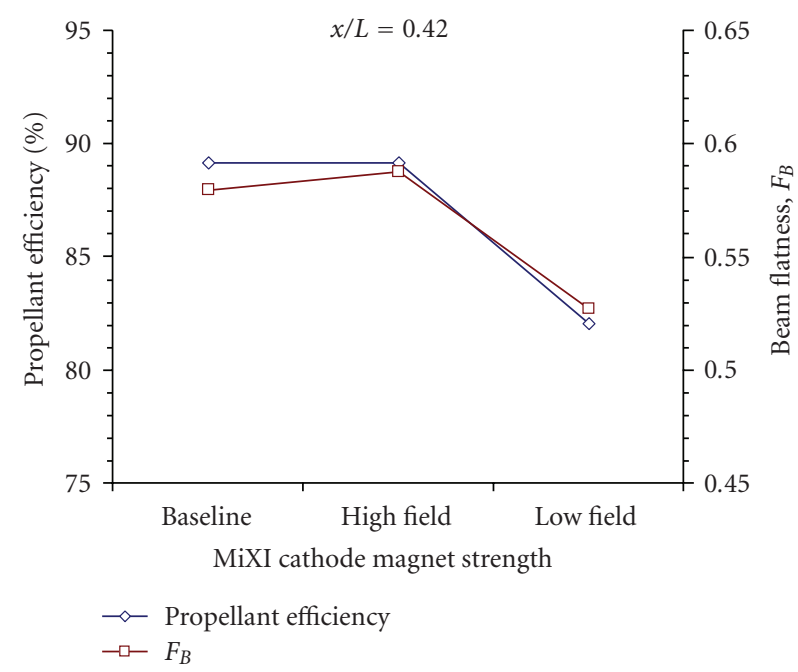

(a)

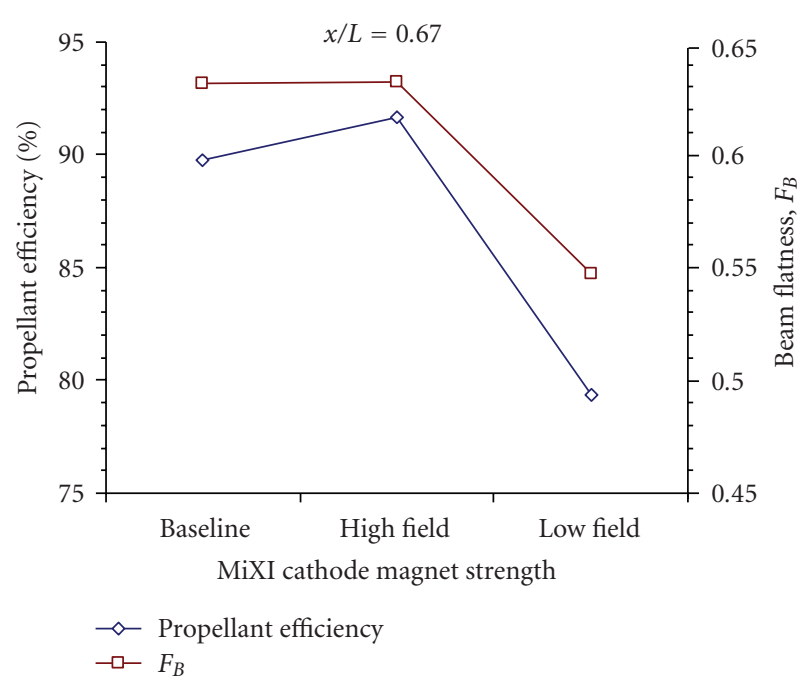

(b)

FIGURE 7: Anticipated MiXI thruster performance for different MiXI cathode magnet strengths. Results generated by DC-ION model.

should not dramatically change the discharge performance. Depending on cathode location, the filament will likely provide a similar but different beam profile when compared with a hollow cathode (possibly flatter due to the more omnidirectional electron emission behavior of the cathode, but similar due to the relatively high average magnetic field strength within the MiXI discharge).

\subsubsection{Vacuum facility}

The experiment was conducted in the $2 \mathrm{~m}$ diameter vacuum chamber (see Figure 10) outfitted with 3 diffusion pumps and 2 cryopumps, as well as several mechanical pumps. In these experiments, only the diffusion pumps were used. The vacuum achievable with these pumps was between $1 \times 10^{-6}$ 


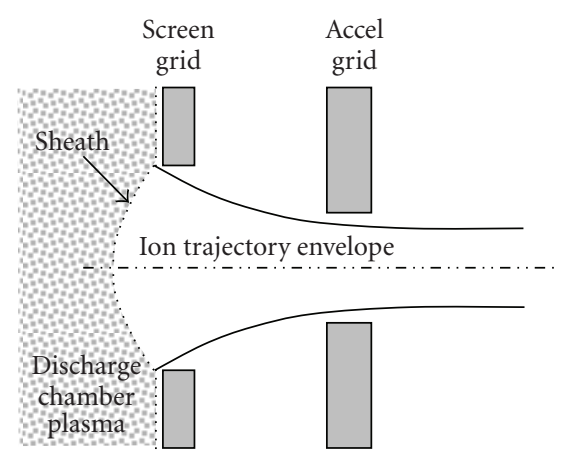

(a)

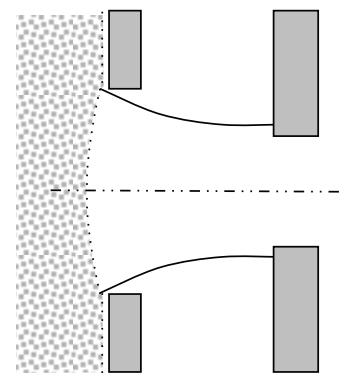

(b)

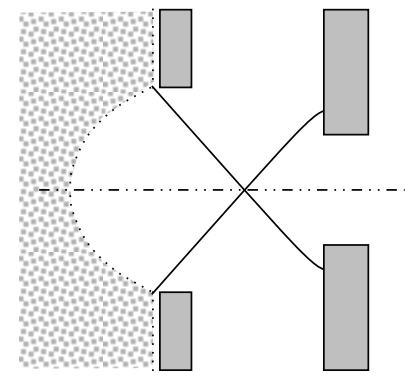

(c)

FIGURE 8: Screen hole plasma sheath and ion trajectory envelope for constant upstream plasma conditions. (a) Nominal $\left(V_{\text {xo }}>V_{T}>V_{\text {foc }}\right)$, (b) direct impingement $\left(V_{T}<V_{\text {foc }}\right)$, (c) cross-over impingement $\left(V_{T}>V_{\mathrm{xo}}\right)$.

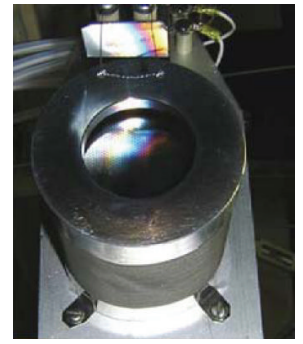

(a)

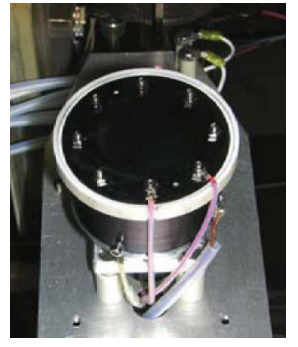

(b)

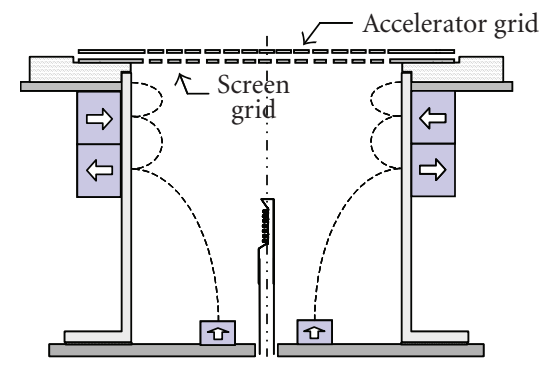

(c)

FIgURe 9: MiXI-II thruster with plasma screen ((a), note filament neutralizer) and without plasma screen (b). A schematic of the MiXI 3-ring configuration is shown at (c).

and $5 \times 10^{-6}$ Torr depending on propellant flow rate. The MiXI-II thruster was pointed along the chamber axis towards the far end of the chamber, and was offset horizontally $0.15 \mathrm{~m}$ from the centerline.

\subsection{Experimental analysis of low thrust regime}

During each test, the discharge current, $J_{D}$, was set at a specified value while the total voltage between the two grids, $V_{T}$, was varied. Discharge voltage, $V_{D}$, was held at $25 \mathrm{~V}$ for all tests. To simulate a steady discharge with a constant ion density, the discharge current was held within 5\% of the reported average value over the entire range of total extraction voltage. As expected, with increasing total voltage, $J_{B}$ reaches a near-constant maximum value while $J_{A}$ reaches a near-constant minimum value for all the discharge current levels. The data in Figure 11 show that the beam "focuses" at increasing voltages for increasing discharge current level and that cross-over impingement is not achieved for the densities and voltages investigated.

This experiment was primarily aimed at the lowdischarge current regime since potential missions considering the MiXI thruster, such as NASA's exoplanet finding missions and the Space Interferometer Mission (SIM), desire that MiXI produces thrust over a large thrust range, $\mathrm{mN}$ to sub-mN levels. Therefore, test cases were conducted with very low discharge currents (i.e., $<20 \mathrm{~mA}$ ). Two test cases at

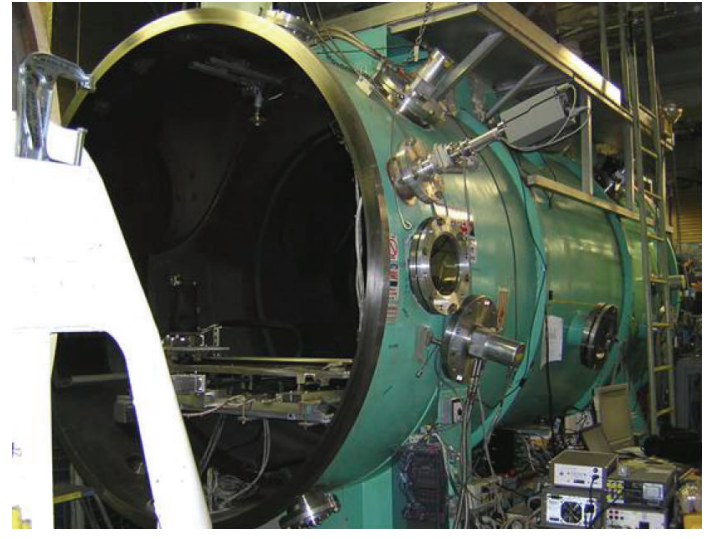

FIGURE 10: JPL's building 149 vacuum chamber ( $2 \mathrm{~m}$ diameter).

low discharges are shown in Figure 12. For an average $J_{D}$ of $17 \mathrm{~mA}$, the focusing voltage, $V_{\text {foc }}$, at which $J_{A}$ and $J_{B}$ become approximately constant occurs at around 400 to $450 \mathrm{~V}$. For the $13 \mathrm{~mA}$ case, this appears to occur at around 450 to $50 \mathrm{~V}$, although it is difficult to tell the exact point due to the inconsistencies in the data trend (especially the $J_{A}$ curve). During data acquisition, the low $J_{D}$ test cases exhibited more noticeable fluctuations in $J_{B}$ and $J_{A}$ than those conducted at higher values of $J_{D}$, so approximate values are reported. Further examination is needed to explain why the focusing 


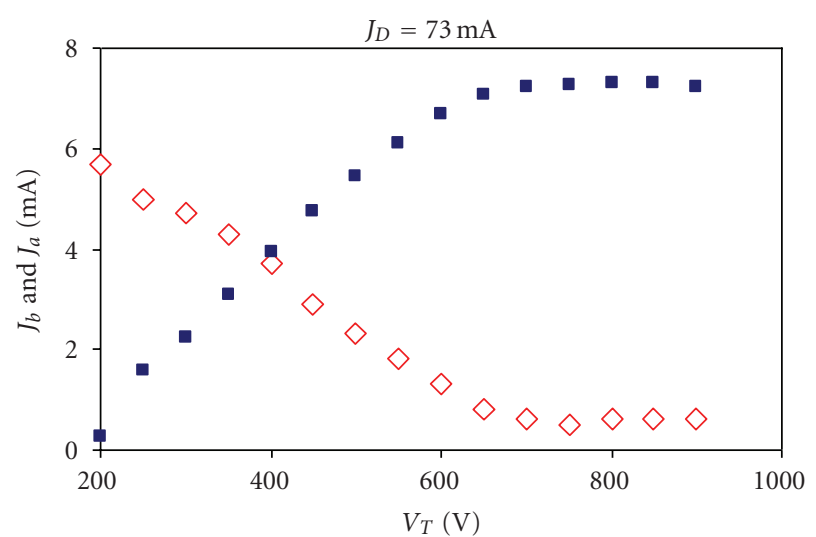

- $J_{b}$

$\diamond J_{a}$

(a)

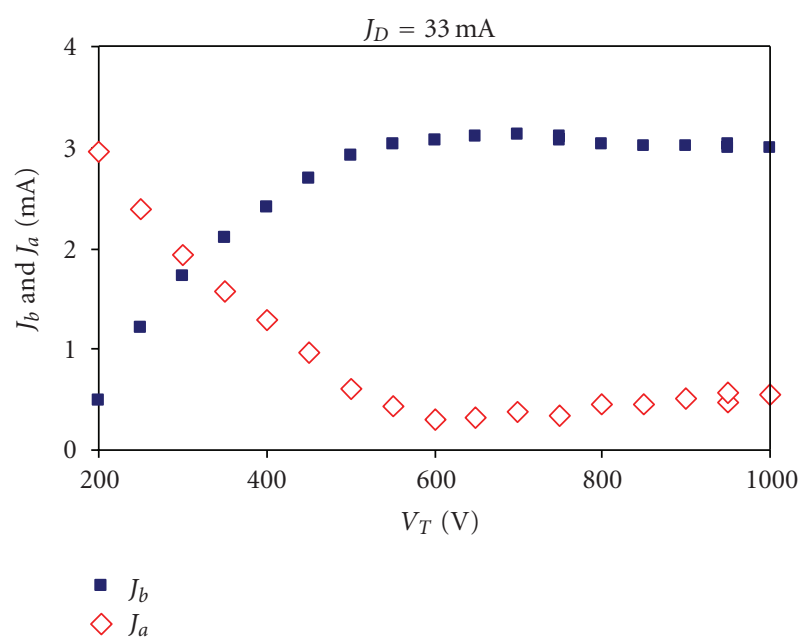

(c)

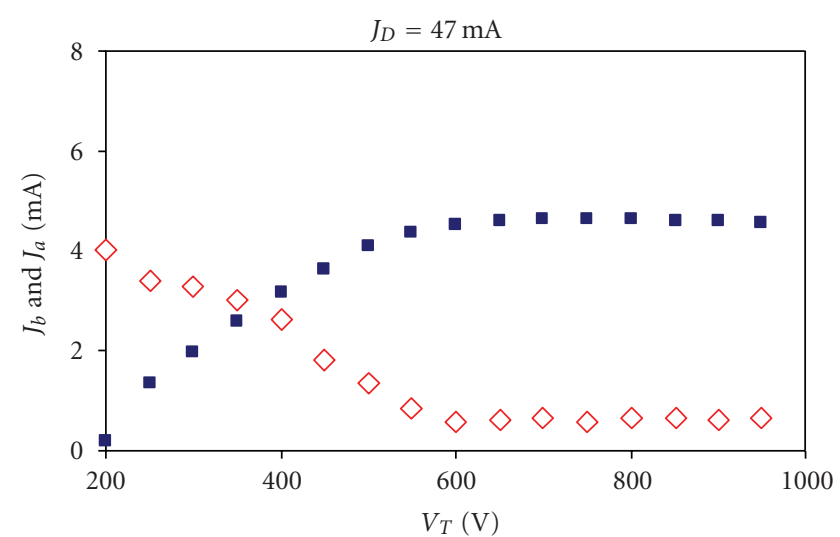

- $J_{b}$

$\diamond J_{a}$

(b)

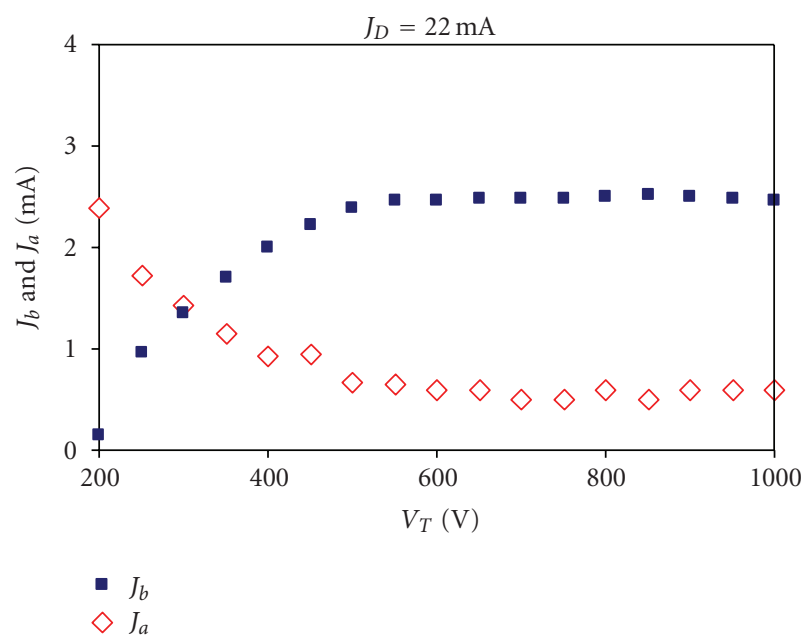

(d)

Figure 11: $J_{B}$ and $J_{A}$ for low $J_{D}$ values for MiXI-II.

voltage and maximum beam current are greater for the $13 \mathrm{~mA}$ versus the $17 \mathrm{~mA}$ case, which is contrary to the expected trends from Figure 11. The increase in $J_{A}$ at higher voltages for the $17 \mathrm{~mA}$ case does not appear to signify crossover impingement due to the constantly increasing levels of beam current.

The results in Figures 11 and 12 follow the expected space-charge current limited operation where the "focusing voltage" increases with upstream ion density (or in this case, discharge current). This is apparent in Figures 13 and 14 where all data are plotted on the same scale for comparison with the computational data in the following section.

\subsection{Computational analysis of low thrust regime}

The 2D ion optics model, CEX2D, was used to simulate single beamlets of the MiXI grid geometry over a range of upstream plasma densities and extraction voltages [8]. For the calculations performed herein, the model requires grid geometry, grid voltages, upstream plasma density, upstream electron temperature, and downstream electron temperature. The plasma density is inferred as discussed below while the electron temperatures are assumed equal to experimental values from similar experiments [4]. As shown in Figure 15, the CEX2D model outputs example ion trajectories that demonstrate the transition from directimpingement to focused beamlet conditions.

The CEX2D model demonstrates the behavior of a single beamlet. To predict the total beam current, $J_{B}$, from CEX2D's beamlet current density, $j_{B}$, we assumed an average beam flatness, $F_{B}$, over the MiXI grid area, such that

$$
J_{B} \approx F_{B} \cdot j_{B} \pi r_{\text {grid }}^{2},
$$

where $j_{B}$ is the current density over the "hexagonal" computational area simulated by CEX2D [11]. We used a relatively low value for beam flatness of only 0.52 , a minimum of the beam flatness from previous MiXI beam profiles at low 


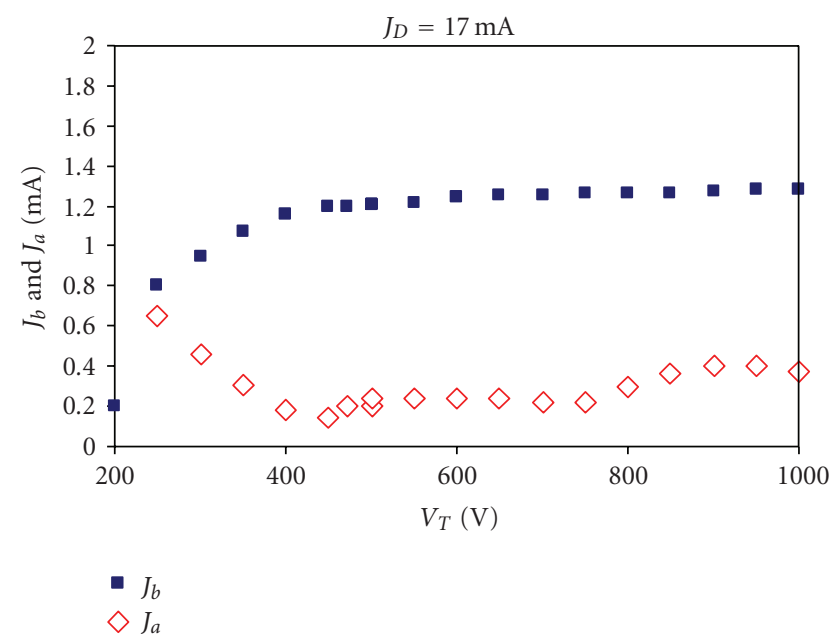

(a)

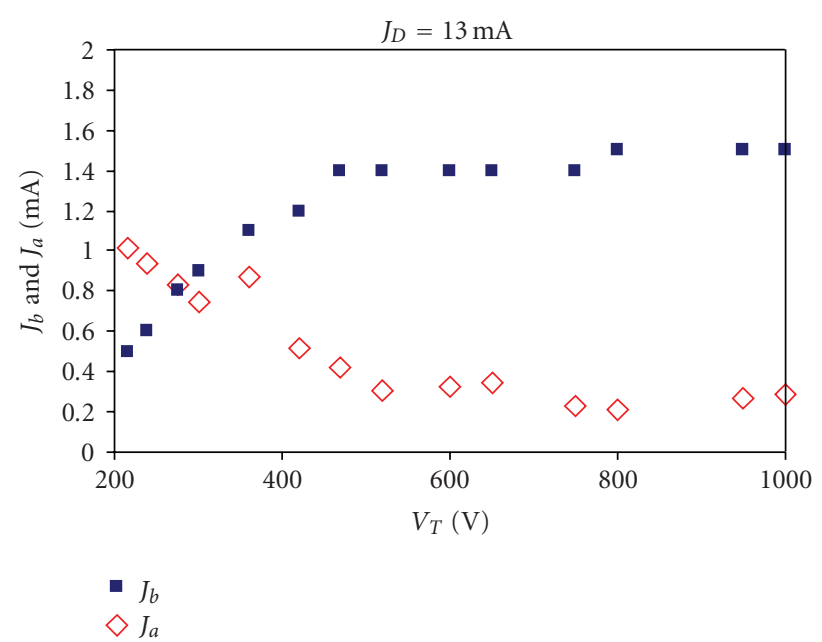

(b)

Figure 12: $J_{B}$ and $J_{A}$ for very low $J_{D}$ values for MiXI-II.

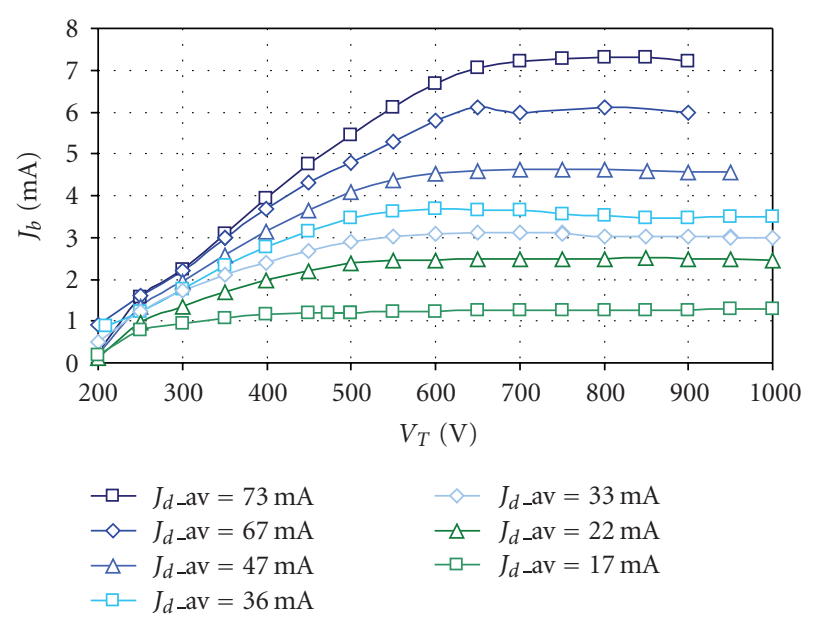

Figure 13: Summary of beam current data.

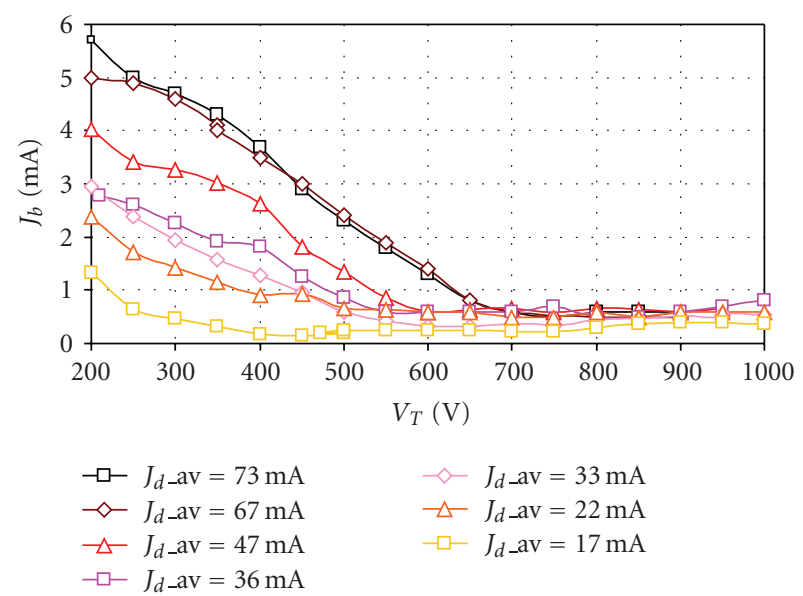

FIGURE 14: Summary of accel grid data. discharge currents. For this first-order analysis, the beamlets are assumed to exhibit identical focusing voltages as the beamlet modeled by CEX2D (a more complete analysis would include consideration of the focusing behavior at the range of densities typically found across the grid surface). Therefore, the average upstream plasma density assumed inside the plasma discharge is obtained by multiplying the beam flatness by the plasma density that is used by CEX2D to provide agreement with the experimentally measured value of total beam current per the above equation. Therefore, the upstream plasma densities are inferred from the measured total beam current and are not directly measured. For these approximations, Figure 16 shows the expected beam current for a range of extraction voltages for different average upstream plasma densities. As expected, these results yield a narrow range of focusing voltages from 350 to $450 \mathrm{~V}$ due to the single beamlet approximation, which is independent of the radial distribution of densities upstream for an actual grid set. For the same reasons, the accel grid current from the computational results quickly drops to zero over a small voltage range, as shown in Figure 17.

\subsection{Discussion of low-thrust analysis of extraction grids}

\subsubsection{Discussion of experimental results}

For the experimental results, beam focusing is the region of simultaneous decrease in $J_{A}$ and an increase in $J_{B}$; and the value at which these currents both reach a near zero slope is the focusing voltage, $V_{\text {foc }}$. The focusing voltages and maximum beam currents for the different discharge currents used in the experimental tests are shown in Figure 18. The general trend of increased focusing voltage and beam current for higher discharge current is expected as discussed in Section 3 and in $[2,9]$. Further investigation is needed to explain the increase in focusing voltage and 


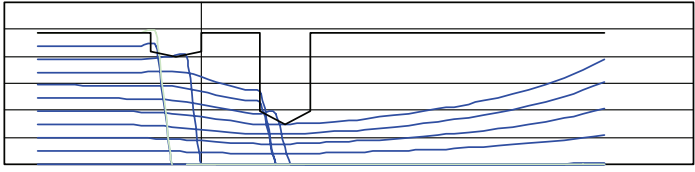

(a)

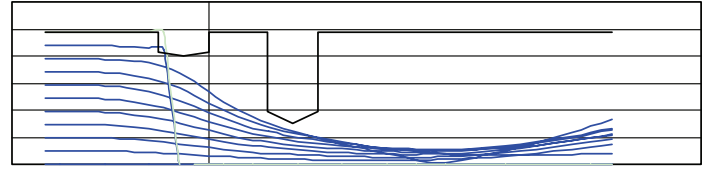

(b)

Figure 15: Example of beam focusing for $V_{B}-V_{A}=250 \mathrm{~V}$ (a) and for $V_{B}-V_{A}=550 \mathrm{~V}$ (b). Bottom of figures are the centerline of a axisymmetric beamlet. Results produced by CEX2D.
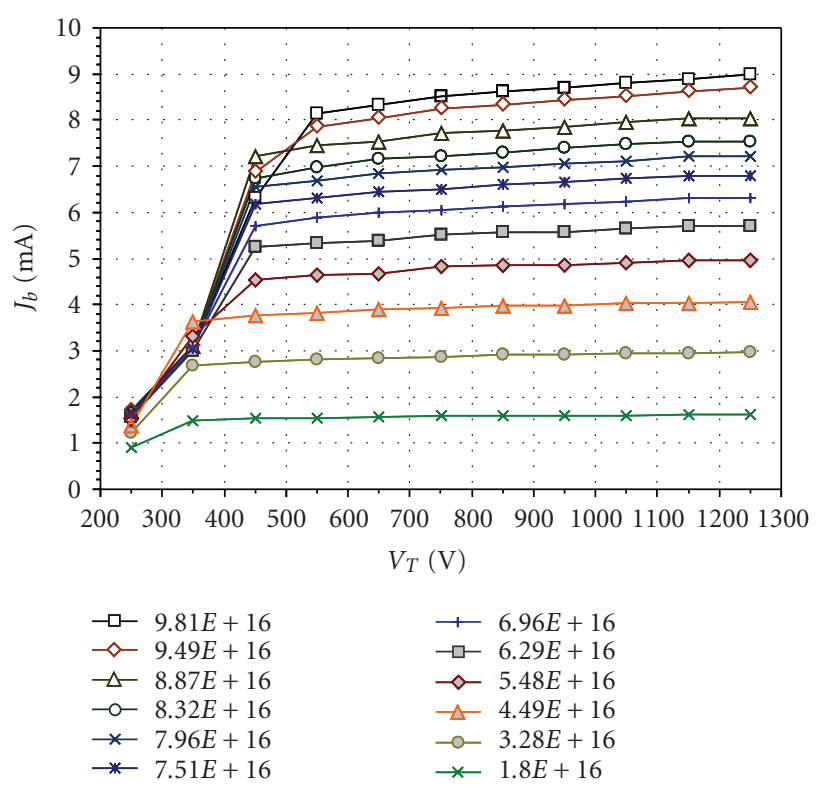

Figure 16: Beam current versus accelerating voltage for averaged discharge plasma densities $\left(\mathrm{m}^{-3}\right)$. Results averaged from CEX2D beamlet results.

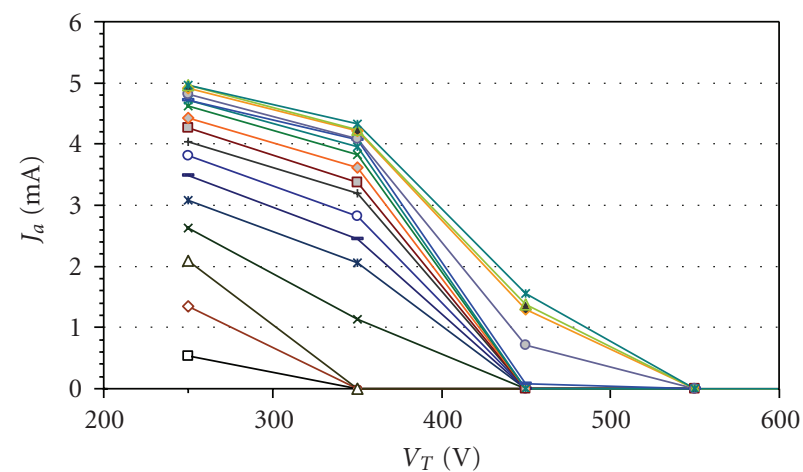

$$
\begin{aligned}
& \neg-5.1 E+16 \quad \checkmark-4.61 E+16 \\
& \checkmark 5.08 E+16 \quad \rightarrow 4.33 E+16 \\
& \triangle 5.05 E+16 \quad \rightarrow \quad 4.14 E+16 \\
& \leftarrow 5.02 E+16 \quad-3.91 E+16 \\
& \text { * } 4.98 E+16 \quad-\quad-3.62 E+16 \\
& -4.93 E+16 \quad \longrightarrow 2.85 E+16 \\
& \text { - } 4 \text { - } 4.88 E+16 \text { - } 2.33 E+16 \\
& \rightarrow 4.8 E+16 \quad \rightarrow-9.36 E+15 \\
& \rightarrow \square-4.72 E+16
\end{aligned}
$$

Figure 17: Accel grid current versus total voltage for averaged discharge plasma densities $\left(\mathrm{m}^{-3}\right)$. Accel current values are zero from 600 to $1300 \mathrm{~V}$. Results averaged from CEX2D beamlet results.

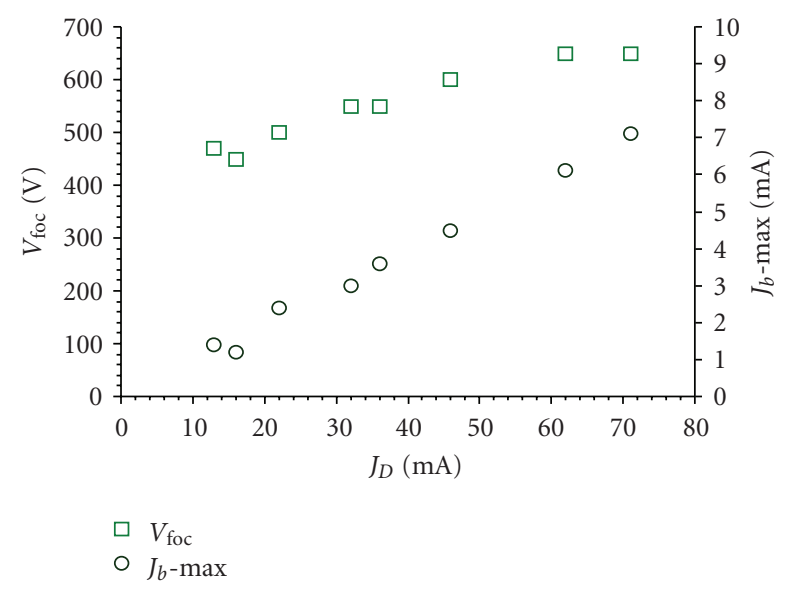

FIGURE 18: Focusing voltage and maximum beam current versus discharge currents used in experimental tests.

beam current at the lowest discharge current (13 mA) value. During the experimental tests, the extraction voltage was increased beyond $V_{\text {foc }}$ in an attempt to achieve cross-over impingement; however, this point was not reached since increasing the beam voltage beyond $1000 \mathrm{~V}$ caused the grids to short for these tests. The relatively high ratio of $J_{A} / J_{B}$ measured suggests that low-thrust operation may lead to decreased grid life due to direct impingement. Additional efforts are needed to determine to what level the high accel current is caused by variation in upstream plasma density (low-beam flatness) which can lead to cross-over impingement at the high radial gridlet locations. Also, longterm erosion predictions and assessments will be needed to assess grid life for given mission profiles.

\subsubsection{Comparison of experimental results and computational results}

The computational results produce qualitative trends similar to those found in the measured data; however, the transition to $V_{\text {foc }}$ occurs much more sharply in the computational data. This is due to the simplifying assumption in the computational analysis that characteristics of one beamlet represent the behavior of all beamlets. For actual ion thrusters, the radial variation in the ion density in the discharge chamber leads to a variation in focusing voltage across the grid surface. Thus the transition to focused operation should be more gradual as seen in the measured data. Also, the prediction of zero accel current is not realized 


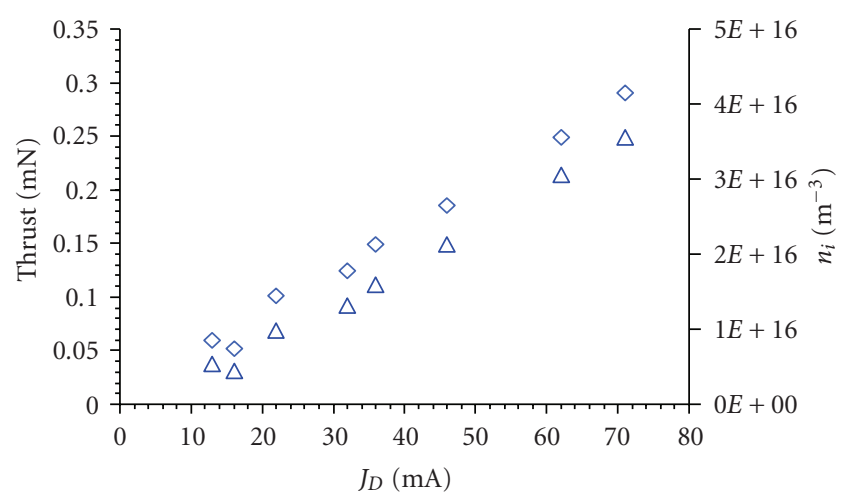

$\triangle$ Thrust

$\diamond$ Density

FIGURE 19: Approximate thrust and average discharge plasma density versus discharge current.

in the measured data. After focusing occurs, we see a nominal accel current of at least $0.3 \mathrm{~mA}$ in the measured data, which is likely an effect of the nonuniform ion density, variation in the MEMS-fabricated hole sizes, and impingement of chargeexchange ions on the downstream surface of the accel grid. For these reasons, this first-order computational analysis also under-predicts the focusing voltage and over-predicts the rate at which the beam focuses. However, these effects do not greatly affect the total beam current predicted by the model and by comparing the maximum beam current values from the experimental (Figure 13) and computational data (Figure 16) we can approximate the average discharge plasma densities for the discharge currents used in the experiment. This comparison yields the correlation of average discharge plasma density and discharge current shown in Figure 19. Also in Figure 19, we approximate the thrust at the focusing voltages for each condition, assuming no beam divergence or double ions losses [4].

In light of the needs of future efforts, it would be helpful for the CEX2D grid model to facilitate the simulation of an entire grid set for a given upstream density profile as opposed to single gridlets. Another possible improvement to the CEX2D model would be to randomize the initial velocity direction of the ions that enter the plasma sheath (currently the model assumes the ions enter the sheath with Bohm velocity parallel to the beamlet axis), this modification would likely give a more realistic value for direct accel grid impingement, that is, nonzero values for focused cases, if a sufficient number of particles are used in the simulation.

\section{CONCLUSIONS}

The computational analysis in Section 2 of this paper suggests that a hollow cathode electron source in the existing MiXI geometry should provide propellant efficiency of almost $90 \%$ and beam flatness of approximately 0.63 . As expected, the cathode provides better performance and beam fla tness at upstream mounting locations. Increasing the cathode magnet strength does not appear to significantly improve the performance; however, a noticeable propellant efficiency loss of up $\sim 12 \%$ is anticipated if the cathode magnet is demagnetized by the cathode heat. These findings are currently under implementation in the lab to guide the positioning of MiXI's miniature hollow cathode inside the discharge. The experimental data of the MiXI-II beam shows that the existing grid set allows thrust levels $\leq 0.03 \mathrm{mN}$ at focused beamlet conditions. As shown by the computational analysis of the beamlets, lower discharge plasma densities (on the order of $\sim 5 \times 10^{15}-5 \times 10^{16} \mathrm{~m}^{-3}$, which are inferred from the experimental and computational results) allow very low total extraction voltage of only $\sim 250-500 \mathrm{~V}$, thus providing the lowest thrust levels thus demonstrated by MiXI. Consequently, the thrust range demonstrated by MiXI in this and other tests is $0.03-1.54 \mathrm{mN}$, assuming no beam divergence or double ion losses. As with many developmental ion thruster efforts, these thrust values are inferred from beam voltage and current; future tests will provide direct thrust measurements. This large continuous thrust range at high efficiency is very attractive to a number of future missions that desire the unique advantages of ion propulsion at the sub-mN-to-mN scale.

\section{NOMENCLATURE}

$F_{B}$ : Beam flatness

$j_{B}$ : Beamlet current density

$J_{a}:$ Total accel grid current

$J_{B}$ : Total beam current

$J_{D}:$ Discharge current

$J_{p}: \quad$ Primary electron current

L: $\quad$ Discharge chamber length

$\dot{m}_{d}$ : Discharge chamber propellant mass flow rate

$n_{i}$ : Ion number density (total)

$n_{o}$ : Neutral atom number density

$r$ : Distance from thruster axis

$T_{p}$ : Primary electron temperature

$V_{A}$ : Accelerator grid voltage

$V_{B}$ : Beam voltage

$V_{D}$ : Discharge voltage

$V_{p}$ : Primary electron voltage

$V_{T}$ : Total voltage (i.e., $V_{B}-V_{A}$ )

$V_{\text {foc }}$ : Voltage below which beamlet transitions to direct impingement operation (Figure 8)

$V_{\mathrm{xo}}$ : Voltage above which beamlet transitions to cross-over operation (Figure 8)

$x$ : Distance between cathode face and grids (Figure 3).

\section{GREEK SYMBOLS}

$\eta_{\mathrm{ud}}$ : Discharge propellant utilization efficiency $\eta_{\text {tot }}:$ Total efficiency

\section{UNITS}

This study uses mks units of the international system (SI)with the exception that energies are frequently given in terms of electron volts $(\mathrm{eVs}) . \mathrm{sccm} \equiv$ standard cubic 
centimeters per minute. For xenon: $1 \mathrm{sccm} \approx 0.09839 \mathrm{mg} / \mathrm{s}$ at STP.

\section{ACKNOWLEDGMENTS}

The authors sincerely thank Lee Johnson of JPL for his support of this task. The research described in this paper was carried out by the Jet Propulsion Laboratory, California Institute of Technology.

\section{REFERENCES}

[1] R. Wirz, J. Mueller, M. Gale, and C. Marrese, "Miniature ion engine for precision formation flying," in Proceedings of the 40th AIAA/ASME/SAE/ASEE Joint Propulsion Conference \& Exhibit, Fort Lauderdale, Fla, USA, July 2004, AIAA-20044115 .

[2] R. Wirz, J. Polk, C. Marrese, and J. Mueller, "Experimental and computational investigation of the performance of a micro-ion thruster," in Proceedings of the 38th AIAA/ASME/SAE/ASEE Joint Propulsion Conference \& Exhibit, Indianapolis, Ind, USA, July 2002, AIAA-2002-3835.

[3] R. Wirz, D. Goebel, C. Marrese, and J. Mueller, "Development of cathode technologies for a miniature ion thruster," in Proceedings of the 39th AIAA/ASME/SAE/ASEE Joint Propulsion Conference \& Exhibit, Huntsville, Ala, USA, July 2003, AIAA2003-4722.

[4] R. Wirz, Discharge plasma processes of ring-cusp ion thrusters, Ph.D. dissertation, Aeronautics, Caltech, Pasadena, Calif, USA, 2005.

[5] M. T. Domonkos, Evaluation of low-current orificed hollow cathodes, Ph.D. thesis, University of Michigan, Ann Arbor, Mich, USA, 1999.

[6] M. T. Domonkos, A. D. Gallimore, and M. J. Patterson,, "An evaluation of hollow cathode scaling to very low power and flow rate," in Proceedings of the 25th International Electric Propulsion Conference, Cleveland, Ohio, USA, August 1997, IEPC-97-189.

[7] M. T. Domonkos, A. D. Gallimore, G. J. Williams Jr., and M. J. Patterson, "Low-current hollow cathode evaluation," in Proceedings of the 35th AIAA/ASME/SAE/ASEE Joint Propulsion Conference \& Exhibit, Los Angeles, Calif, USA, June 1999, AIAA-99-2575.

[8] R. Wirz and D. Goebel, "Ion thruster discharge performance per magnetic field topography," in Proceedings of the 42nd AIAA/ASME/SAE/ASEE Joint Propulsion Conference \& Exhibit, Sacramento, Calif, USA, July 2006, AIAA-2006-4487.

[9] R. Wirz and I. Katz, "Plasma processes of DC ion thruster discharge chambers," in Proceedings of the 41st AIAA/ASME/SAE/ASEE Joint Propulsion Conference \& Exhibit, Tucson, Ariz, USA, July 2005, AIAA-2005-3690.

[10] G. Aston, H. R. Kaufman, and P. J. Wilbur, "Ion beam divergence characteristics of two-grid accelerator systems," AIAA Journal, vol. 16, no. 5, pp. 516-524, 1978.

[11] J. R. Brophy, I. Katz, J. E. Polk, and J. R. Anderson, "Numerical simulations of ion thruster accelerator grid erosion," in Proceedings of the 38th AIAA/ASME/SAE/ASEE Joint Propulsion Conference \& Exhibit, Indianapolis, Ind, USA, July 2002, AIAA-2002-4261. 